\title{
ANN: Model of Back-Propagation Architecture on the Logs Production by Type of Wood
}

\author{
Muhammad Noor Hasan Siregar \\ Universitas Graha Nusantara, Padangsidimpuan, Sumatera Utara \\ noor.siregar@gmail.com
}

\begin{abstract}
Indonesia is rich in forest products. The production forest is a forest area that can be utilized for the community, such as logs, rattan, and some forest plants that have high economic value. This research aims to make the best architectural model by using artificial neural network. The method used is backpropagation algorithm. With this model it will continue to predict the output of logs. Data are sourced from BPS-Statistics Indonesia. Based on the results of research results of logs production using backpropogation method, obtained the result of 3 model architecture (18-18-1, 18-25-1 and 18-18-25-1) that model of architecture 18-25-1 is the best model with $72 \%$ accuracy, MSE: 0.0221670942 and epochs: 660.
\end{abstract}

Keywords: ANN, Logs production, Backpropagation, JST, Prediction

\section{Introduction}

Indonesia is rich in forest products, but not all forests can be harnessed and produced. For forest utilization, the government has established certain forest areas designated as Production Forest. The production forest is a forest area whose results can be utilized for the community, such as logs, rattan, and some forest plants that have high economic value. Logs that are cut or harvested can be used as raw materials for upstream wood processing production. This production of logs is produced from natural forests through the activities of forest concession companies. Forest Concessionaire Enterprises is a business / legal entity engaged in the field of harvesting of forest products. Based on the source of Indonesia's Forestry Statistics Book, the data of log production in Indonesia for the last 10 years has increased by $314.64 \%$ In 2001 Indonesia's log production amounted to $11,432,501 \mathrm{~m} 3$ and in 2011 amounted to $47,429,335 \mathrm{~m} 3$.

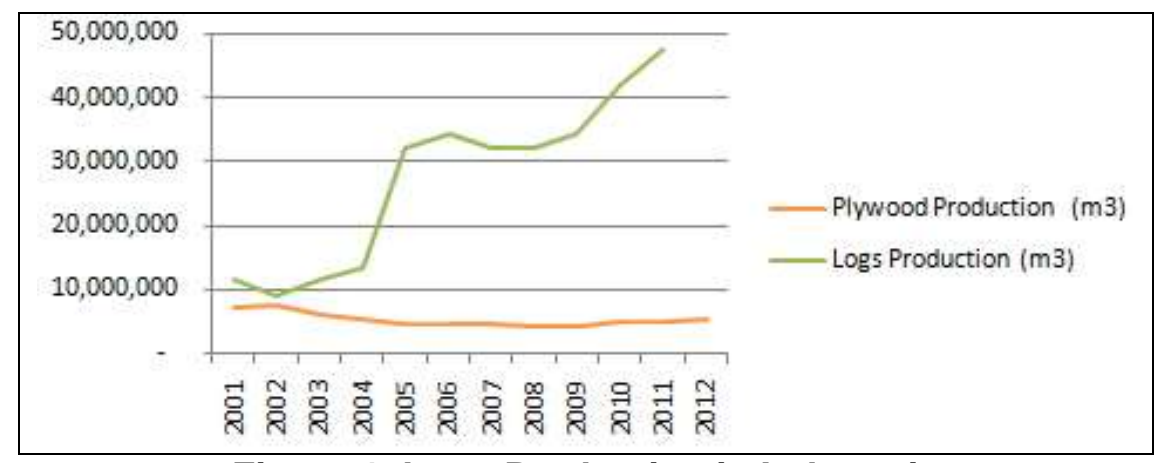

Figure 1. Logs Production in Indonesia

(Source : Indonesia Forest Statistics Book)

Increased production of logs gives some negative impacts. One of them is the destruction of natural ecosystems that encourage the emergence of concerns shortage of industrial raw materials in the future. This study aims to create a prediction model of the total prediction of logs in Indonesia using artificial intelligence. This model can then be used to predict long-term log production so that the government can anticipate and provide solutions to the negative impacts resulting from the production of logs in Indonesia. One 
branch of computer science related to prediction is artificial intelligence. There is a lot of artificial intelligence that deals with predictions.

The Artificial Neural Network is one of the branches of artificial intelligence [1]. Today, the AI is a very important discipline and it includes a number of well-recognized and mature areas including Expert Systems [2-4], Fuzzy Logic [5-8], Genetic Algorithms [9-11], Language Processing, Logic Programming, Planning and Scheduling, Neural Networks and Robotics [12]. There are many techniques that can be used for the implementation of the Neural Network Tirua one of the methods used is Backpropagation [2-6]. Backpropogation is one of the artificial neural network algorithms that is often used to solve complex problems related to input identification, prediction, pattern recognition, and so on. Repeated training will result in a network that responds correctly to all its inputs.

\section{Rudimentary}

\subsection{Artificial Intelegence}

AI is a field of study based on the premise that intelligent thought can be regarded as aform of computation - one that can be formalized and ultimately mechanized. To achievethis, however, two major issues need to be addressed. The first issue is knowledge representation, and the second is knowledge manipulation [1].

\subsection{Artificial Neural Networks (NN)}

Artificial Neural Network (ANN) is a computational model, which is based on Biological Neural Network. Artificial Neural Network is often called as Neural Network(NN) (See Figure 1). From Figure 1, to build artificial neural network, artificial neurons, also called as nodes, are interconnected [13,14]. An artificial neuron is an abstraction of biological neurons and the basic unitin an ANN [15,16]. The Artificial Neuron receives one or more inputs and sums them to produce an output[17,18]. After successful training, user can give unlabeled data to be classified.

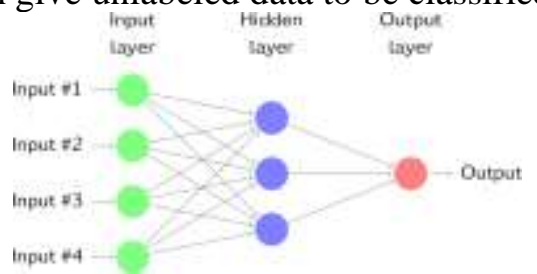

Figure 1. Artificial Neural Network Model

\subsection{Architecture of Backpropogation}

The back-propagation learning algorithm (BPLA) has become famous learning algorithms among ANNs. In the learning process, to reduce the in accuracy of ANNs, BPLAs use the gradient decent search method to adjust the connection weights. The structure of a backpropagation ANN is shown in Figure 2[19]. Each of these layers must be either of the following:

1. Input Layer - This layer holds the input for thenetwork

2. Output Layer - This layer holds the output data, usually an identifier for the input.

3. Hidden Layer - This layer comes between the inputlayer and the output layer[20]. 


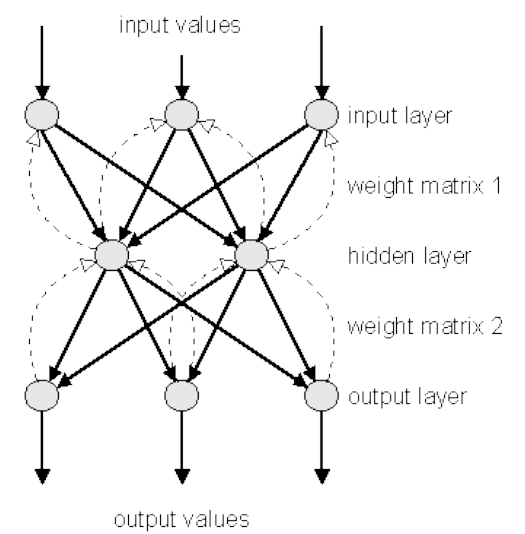

Figure 2. Back-propogation ANN

\subsection{Backpropagation Neural Network}

Phases in Backpropagation Techniquealgorithm can bedivided into two phases: propagation and weight update.

Phase 1: Propagation

Each propagation involves the following steps:

1. Forward propagation of a training pattern's input is given through the neural network in order to generate the propagation's output activations.

2. Back propagation of the output activations propagation through the neural network using the training pattern's targetin order to generate the deltas of all output and hidden neurons.

Phase 2: Weight Update

For each weight-synapse:

1. Multiply its input activation and output delta to get thegradient of the weight.

2. Bring the weight in the direction of the gradient byadding a ratio of it from the weight. [21,22].

\section{Research and Methodology}

\subsection{Research Framework}

A framework of research work used in solving this research problem.

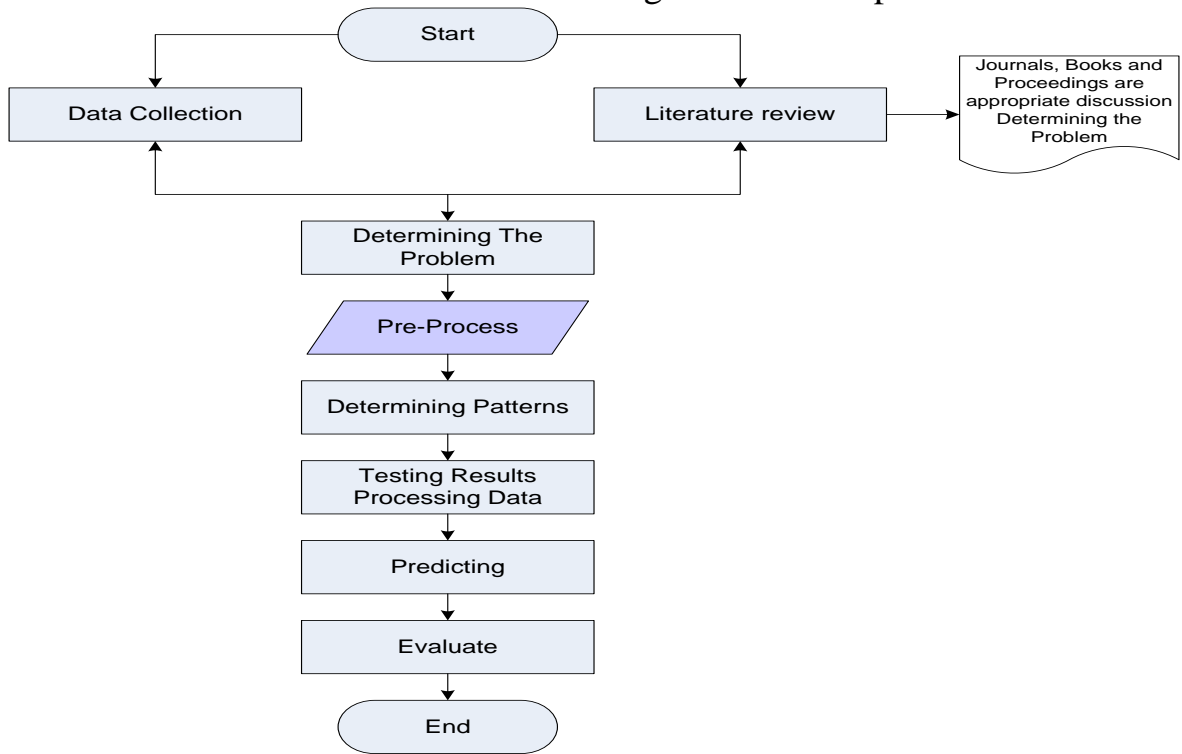

Figure 3. Research Framework 
Based on the framework in the picture above, each step can be described as follows :

1. Collecting Data

2. Library Studies

3. Identifying Problems

4. Preprocess

5. Determining the Model

6. Testing Results Data Processing

7. Predicting

8. Evaluate the End

\subsection{Input Data}

The data used in this research is round wood production data by type obtained from BPS Statistic Indonesia (2004-2015). Log production data can be seen in the following table:

Table 1. Logs Production by Type of Wood (M3)

\begin{tabular}{|c|c|c|c|c|c|c|c|c|c|c|c|c|}
\hline $\begin{array}{l}\text { Type of } \\
\text { Logs }\end{array}$ & 2004 & 2005 & 2006 & 2007 & 2008 & 2009 & 2010 & 2011 & 2012 & 2013 & 2014 & 2015 \\
\hline Agathis & 32134 & 29888 & 1612 & 26132 & 18121 & 6034 & 5853 & 6380 & 4707 & 453 & 453 & 2173 \\
\hline Bakau & 290475 & 213291 & 155582 & 52913 & 55558 & 110205 & 160989 & 0 & 0 & 139521 & 110144 & 14165 \\
\hline Bangkirai & 48776 & 64733 & 66136 & 73207 & 77127 & 77818 & 82063 & 99244 & 112141 & 44244 & 71864 & 112541 \\
\hline Benuang & 14861 & 8029 & 6655 & 9549 & 39945 & 36450 & 36109 & 0 & 5122 & 6098 & 18223 & 15145 \\
\hline Damar & 2777 & 3543 & 1625 & 3830 & 2409 & 1491 & 525 & 0 & 0 & 0 & 0 & 0 \\
\hline Duabanga & 32393 & 0 & 0 & 0 & 0 & 0 & 0 & 0 & 30 & 14 & 14 & 35 \\
\hline Indah & 72980 & 57799 & 45209 & 108083 & 85434 & 59699 & 45307 & 48094 & 41466 & 17959 & 23307 & 34300 \\
\hline Jelutung & 22226 & 1201 & 18580 & 24539 & 24813 & 17431 & 21340 & 0 & 16 & 70 & 471 & 777 \\
\hline Kapur & 307602 & 323635 & 390958 & 261523 & 281591 & 268621 & 250500 & 209827 & 233432 & 31878 & 22486 & 78972 \\
\hline Kruing & 242706 & 372573 & 308901 & 375459 & 372044 & 369933 & 342897 & 168467 & 255099 & 272201 & 262685 & 327803 \\
\hline Meranti & 4135592 & 5049694 & 4377991 & 4460637 & 4231197 & 4062671 & 4385510 & 4091990 & 3160592 & 3082766 & 3742865 & 4169157 \\
\hline Mersawa & 20103 & 14957 & 12675 & 22761 & 106304 & 105334 & 100886 & 3657 & 6675 & 5659 & 9775 & 11238 \\
\hline Nyatoh & 31434 & 26345 & 23587 & 53551 & 41595 & 39141 & 35449 & 22337 & 18460 & 8904 & 10566 & 16272 \\
\hline Palapi & 17598 & 15176 & 20522 & 54185 & 35767 & 15756 & 7222 & 0 & 802 & 292 & 2585 & 5159 \\
\hline Ramin & 81127 & 65393 & 81587 & 92965 & 92425 & 67707 & 31583 & 35256 & 28400 & 0 & 0 & 0 \\
\hline Resak & 3703 & 6045 & 4548 & 8411 & 7458 & 6756 & 4822 & 246 & 2197 & 2019 & 1317 & 3324 \\
\hline Lainnya & 1117565 & 945863 & 909309 & 1061444 & 1040050 & 1237864 & 960548 & 948357 & 849772 & 753926 & 651929 & 513774 \\
\hline
\end{tabular}

Source: BPS-Statistic Indonesia

In table 1, it can be explained that, the input value consists of 18 variables and 1 target value. The input value consists of log type (X1-X18) and the target value consists of the total number of logs production. In the process of selecting the best architectural model using backpropagation, the data is normalized first using the formula :

$x^{\prime}=\frac{0.8(x-a)}{b-a}+0.1$

(1)

After the data is normalized, the process of selecting the best architecture model with backpropogation is divided into 2 parts, namely: data traning and data testing. The results of normalization of data can be seen in the following table:

Table 2. DataTraining After Normalization

\begin{tabular}{|lc|c|c|c|c|c|}
\hline \multicolumn{1}{|c|}{ Types of logs } & $\mathbf{2 0 0 4}$ & $\mathbf{2 0 0 5}$ & $\mathbf{2 0 0 6}$ & $\mathbf{2 0 0 7}$ & $\mathbf{2 0 0 8}$ & $\mathbf{2 0 0 9}$ \\
\hline Agathis & 0,1051 & 0,1047 & 0,1003 & 0,1041 & 0,1029 & 0,1010 \\
\hline Bakau & 0,1460 & 0,1338 & 0,1246 & 0,1084 & 0,1088 & 0,1175 \\
\hline Bangkirai & 0,1077 & 0,1103 & 0,1105 & 0,1116 & 0,1122 & 0,1123 \\
\hline Benuang & 0,1024 & 0,1013 & 0,1011 & 0,1015 & 0,1063 & 0,1058 \\
\hline Damar & 0,1004 & 0,1006 & 0,1003 & 0,1006 & 0,1004 & 0,1002 \\
\hline Duabanga & 0,1051 & 0,1000 & 0,1000 & 0,1000 & 0,1000 & 0,1000 \\
\hline Indah & 0,1116 & 0,1092 & 0,1072 & 0,1171 & 0,1135 & 0,1095 \\
\hline Jelutung & 0,1035 & 0,1002 & 0,1029 & 0,1039 & 0,1039 & 0,1028 \\
\hline
\end{tabular}




\begin{tabular}{|l|l|l|l|l|l|l|} 
Kapur & 0,1487 & 0,1513 & 0,1619 & 0,1414 & 0,1446 & 0,1426 \\
\hline Kruing & 0,1385 & 0,1590 & 0,1489 & 0,1595 & 0,1589 & 0,1586 \\
\hline Meranti & 0,7552 & 0,9000 & 0,7936 & 0,8067 & 0,7703 & 0,7436 \\
\hline Mersawa & 0,1032 & 0,1024 & 0,1020 & 0,1036 & 0,1168 & 0,1167 \\
\hline Nyatoh & 0,1050 & 0,1042 & 0,1037 & 0,1085 & 0,1066 & 0,1062 \\
\hline Palapi & 0,1028 & 0,1024 & 0,1033 & 0,1086 & 0,1057 & 0,1025 \\
\hline Ramin & 0,1129 & 0,1104 & 0,1129 & 0,1147 & 0,1146 & 0,1107 \\
\hline Resak & 0,1006 & 0,1010 & 0,1007 & 0,1013 & 0,1012 & 0,1011 \\
\hline Lainnya & 0,2771 & 0,2498 & 0,2441 & 0,2682 & 0,2648 & 0,2961 \\
\hline Rimba Campuran & 0,3668 & 0,3490 & 0,3338 & 0,3873 & 0,3451 & 0,2979 \\
\hline
\end{tabular}

In table 2, input data is log production (2004-2009) where the input value (X1-X18) and target value (logs production in 2009)

Table 3. Data Testing After Normalization

\begin{tabular}{|l|c|c|c|c|c|c|}
\hline \multicolumn{1}{|c|}{ types of logs } & $\mathbf{2 0 1 0}$ & $\mathbf{2 0 1 1}$ & $\mathbf{2 0 1 2}$ & $\mathbf{2 0 1 3}$ & $\mathbf{2 0 1 4}$ & $\mathbf{2 0 1 5}$ \\
\hline Agathis & 0,1011 & 0,1012 & 0,1009 & 0,1001 & 0,1001 & 0,1004 \\
\hline Bakau & 0,1294 & 0,1000 & 0,1000 & 0,1255 & 0,1201 & 0,1026 \\
\hline Bangkirai & 0,1150 & 0,1181 & 0,1205 & 0,1081 & 0,1131 & 0,1205 \\
\hline Benuang & 0,1066 & 0,1000 & 0,1009 & 0,1011 & 0,1033 & 0,1028 \\
\hline Damar & 0,1001 & 0,1000 & 0,1000 & 0,1000 & 0,1000 & 0,1000 \\
\hline Duabanga & 0,1000 & 0,1000 & 0,1000 & 0,1000 & 0,1000 & 0,1000 \\
\hline Indah & 0,1083 & 0,1088 & 0,1076 & 0,1033 & 0,1043 & 0,1063 \\
\hline Jelutung & 0,1039 & 0,1000 & 0,1000 & 0,1000 & 0,1001 & 0,1001 \\
\hline Kapur & 0,1457 & 0,1383 & 0,1426 & 0,1058 & 0,1041 & 0,1144 \\
\hline Kruing & 0,1626 & 0,1307 & 0,1465 & 0,1497 & 0,1479 & 0,1598 \\
\hline Meranti & 0,9000 & 0,8465 & 0,6766 & 0,6624 & 0,7828 & 0,8605 \\
\hline Mersawa & 0,1184 & 0,1007 & 0,1012 & 0,1010 & 0,1018 & 0,1021 \\
\hline Nyatoh & 0,1065 & 0,1041 & 0,1034 & 0,1016 & 0,1019 & 0,1030 \\
\hline Palapi & 0,1013 & 0,1000 & 0,1001 & 0,1001 & 0,1005 & 0,1009 \\
\hline Ramin & 0,1058 & 0,1064 & 0,1052 & 0,1000 & 0,1000 & 0,1000 \\
\hline Resak & 0,1009 & 0,1000 & 0,1004 & 0,1004 & 0,1002 & 0,1006 \\
\hline Lainnya & 0,2752 & 0,2730 & 0,2550 & 0,2375 & 0,2189 & 0,1937 \\
\hline Rimba Campuran & 0,2586 & 0,2349 & 0,2137 & 0,1888 & 0,1946 & 0,2048 \\
\hline
\end{tabular}

In table 3, input data is log production (2010-2015) where the input value (X1-X8) and target value (logs production in 2015)

\subsection{Output Data}

The expected result is the selection of an arsitertur model to predict the results of the Logs Production by Type of Wood. The best architectural model is seen from the smallest minimum error rate. In this study, the minimum error used is: $(0,001-0,009$ or $(-0,001)-(-$ 0,009): True) and (>0,009: False).

\section{Results and Discussion}

\subsection{Model Arsitektur 18-18-1}

Here are the results of architectural model calculations 18-18-1 (18 inputs, 18 hidden hidden neurons, 1 output) using matlab software.

Table 4. Data Training Backpropagation (18-18-1)

\begin{tabular}{|c|l|l|l|c|c|}
\hline \multirow{2}{*}{ No } & \multirow{2}{*}{ types of logs } & \multirow{2}{*}{ Target } & \multicolumn{3}{|c|}{ ANN 18-18-1 } \\
\cline { 4 - 6 } & & & Output & Error & SSE \\
\hline 1 & Agathis & 0,1010 & 0,1206 & $-0,020$ & 0,0003858891 \\
\hline 2 & Bakau & 0,1175 & 0,1431 & $-0,026$ & 0,0006574467 \\
\hline 3 & Bangkirai & 0,1123 & 0,1176 & $-0,005$ & 0,0000277903 \\
\hline
\end{tabular}




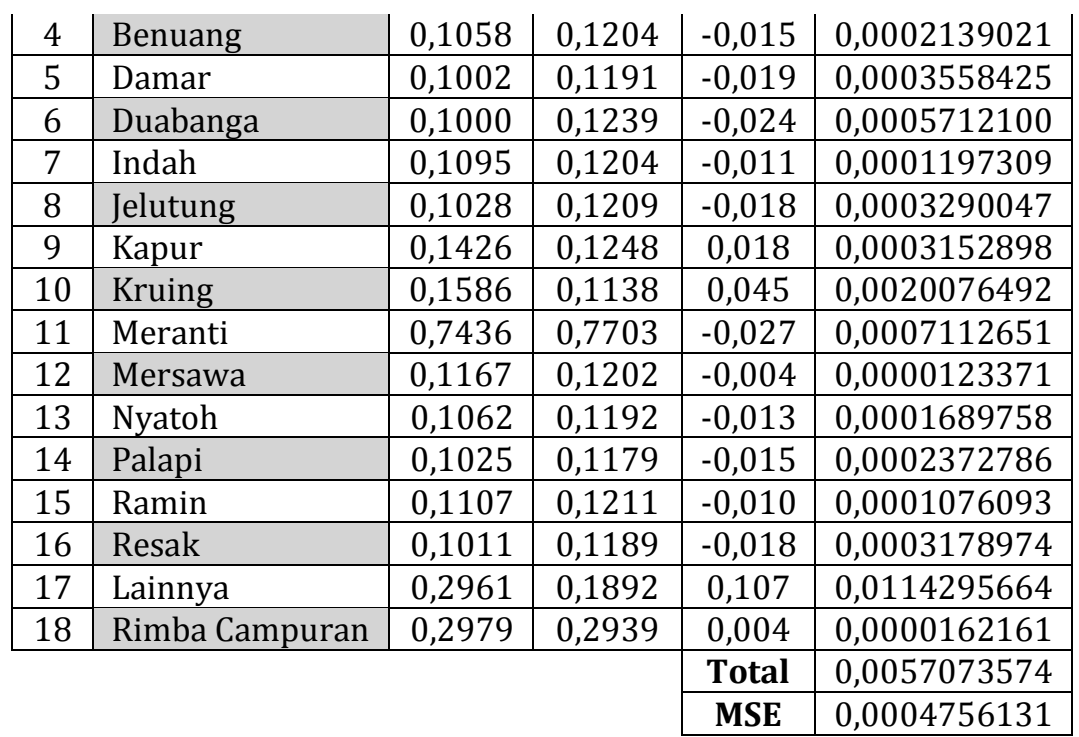

Table 5. Data Testing Backpropagation (18-18-1)

\begin{tabular}{|c|l|c|c|c|c|c|}
\hline \multirow{2}{*}{ No } & \multirow{2}{*}{ types of logs } & \multirow{2}{*}{ Target } & \multicolumn{3}{|c|}{ ANN 18-18-1 } & \multirow{2}{*}{ Result } \\
\cline { 4 - 6 } & & Output & Error & SSE & \\
\hline 1 & Agathis & 0,1004 & 0,1196 & $-0,019$ & 0,0003687784 & FALSE \\
\hline 2 & Bakau & 0,1026 & 0,1414 & $-0,039$ & 0,0015066847 & FALSE \\
\hline 3 & Bangkirai & 0,1205 & 0,1206 & 0,000 & 0,0000000050 & TRUE \\
\hline 4 & Benuang & 0,1028 & 0,1249 & $-0,022$ & 0,0004900585 & FALSE \\
\hline 5 & Damar & 0,1000 & 0,1193 & $-0,019$ & 0,0003724900 & FALSE \\
\hline 6 & Duabanga & 0,1000 & 0,1192 & $-0,019$ & 0,0003683949 & FALSE \\
\hline 7 & Indah & 0,1063 & 0,1212 & $-0,015$ & 0,0002232942 & FALSE \\
\hline 8 & Jelutung & 0,1001 & 0,1228 & $-0,023$ & 0,0005133968 & FALSE \\
\hline 9 & Kapur & 0,1144 & 0,1385 & $-0,024$ & 0,0005805215 & FALSE \\
\hline 10 & Kruing & 0,1598 & 0,1492 & 0,011 & 0,0001123064 & FALSE \\
\hline 11 & Meranti & 0,8605 & 0,6976 & 0,163 & 0,0265472014 & FALSE \\
\hline 12 & Mersawa & 0,1021 & 0,1362 & $-0,034$ & 0,0011662209 & FALSE \\
\hline 13 & Nyatoh & 0,1030 & 0,1225 & $-0,020$ & 0,0003814865 & FALSE \\
\hline 14 & Palapi & 0,1009 & 0,1204 & $-0,019$ & 0,0003786488 & FALSE \\
\hline 15 & Ramin & 0,1000 & 0,1212 & $-0,021$ & 0,0004494400 & FALSE \\
\hline 16 & Resak & 0,1006 & 0,1199 & $-0,019$ & 0,0003722445 & FALSE \\
\hline 17 & Lainnya & 0,1937 & 0,1719 & 0,022 & 0,0004762040 & FALSE \\
\hline 18 & Rimba Campuran & 0,2048 & 0,1898 & 0,015 & 0,0002252364 & FALSE \\
\hline & & & Total & 0,0322493524 & \multirow{2}{*}{$6 \%$} \\
\cline { 5 - 6 } & & & MSE & 0,0026874460 & \\
\hline
\end{tabular}

In Table 5, the back-propagation architectural model 18-18-1 has an accuracy of 6\% with MSE: 0.0026874460 and epochs: 3797

\subsection{Model Arsitektur 18-25-1}

Here are the results of architectural model calculations 18-25-1 (18 inputs, 25 hidden hidden neurons, 1 output) using matlab software.

Table 6. Data Training Backpropagation (18-25-1)

\begin{tabular}{|c|l|l|l|c|c|}
\hline \multirow{2}{*}{ No } & \multirow{2}{*}{ types of logs } & \multirow{2}{*}{ Target } & \multicolumn{3}{|c|}{ ANN 18-25-1 } \\
\cline { 4 - 6 } & & & Output & Error & SSE \\
\hline 1 & Agathis & 0,1010 & 0,1072 & $-0,006$ & 0,0000389883 \\
\hline 2 & Bakau & 0,1175 & 0,1229 & $-0,005$ & 0,0000296015 \\
\hline 3 & Bangkirai & 0,1123 & 0,1055 & 0,007 & 0,0000466264 \\
\hline
\end{tabular}




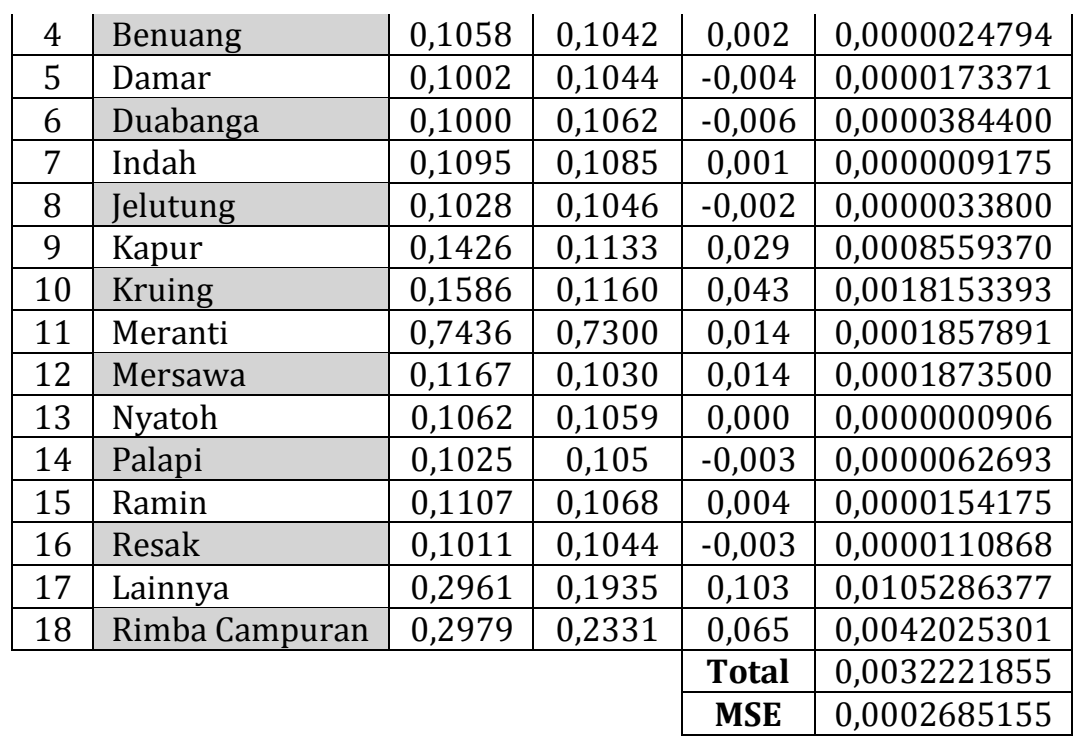

Table 7. Data Testing Backpropagation (18-25-1)

\begin{tabular}{|c|c|c|c|c|c|c|}
\hline & \multirow{2}{*}{ types of logs } & \multirow{2}{*}{ Target } & \multicolumn{3}{|c|}{ ANN 18-25-1 } & \multirow{2}{*}{ Result } \\
\hline NO & & & Output & Error & SSE & \\
\hline 1 & Agathis & 0,1004 & 0,1046 & $-0,004$ & 0,0000176703 & TRUE \\
\hline 2 & Bakau & 0,1026 & 0,1160 & $-0,013$ & 0,0001799900 & FALSE \\
\hline 3 & Bangkirai & 0,1205 & 0,1062 & 0,014 & 0,0002053377 & FALSE \\
\hline 4 & Benuang & 0,1028 & 0,1061 & $-0,003$ & 0,0000111373 & TRUE \\
\hline 5 & Damar & 0,1000 & 0,1043 & $-0,004$ & 0,0000184900 & TRUE \\
\hline 6 & Duabanga & 0,1000 & 0,1042 & $-0,004$ & 0,0000175864 & TRUE \\
\hline 7 & Indah & 0,1063 & 0,1066 & 0,000 & 0,0000001177 & TRUE \\
\hline 8 & Jelutung & 0,1001 & 0,1057 & $-0,006$ & 0,0000308943 & TRUE \\
\hline 9 & Kapur & 0,1144 & 0,1187 & $-0,004$ & 0,0000184385 & TRUE \\
\hline 10 & Kruing & & 0,1185 & 0,041 & 0,0017054810 & FALSE \\
\hline 11 & Meranti & 0,8605 & 0,3470 & 0,514 & 0,2637162646 & FALSE \\
\hline 12 & Mersawa & 0,1021 & 0,1112 & $-0,009$ & 0,0000837221 & TRUE \\
\hline 13 & Nyatoh & 0,1030 & 0,1064 & $-0,003$ & 0,0000117764 & TRUE \\
\hline 14 & Palapi & 0,1009 & 0,1046 & $-0,004$ & 0,0000133876 & TRUE \\
\hline 15 & Ramin & 0,1000 & 0,1062 & $-0,006$ & 0,0000384400 & TRUE \\
\hline 16 & Resak & 0,1006 & 0,1045 & $-0,004$ & 0,0000151604 & TRUE \\
\hline 17 & Lainnya & 0,1937 & 0,1932 & 0,001 & 0,0000002726 & TRUE \\
\hline 18 & Rimba Campuran & 0,2048 & 0,1752 & 0,030 & 0,0008766265 & FALSE \\
\hline & & & & Total & 0,2660051298 & 72\% \\
\hline
\end{tabular}

In Table 7, the back-propagation architectural model 18-25-1 has an accuracy of 72\% with MSE: 0.0221670942 and epochs: 660

\subsection{Model Arsitektur 18-18-25-1}

Here are the results of architectural model calculations 18-18-25-1 (18 inputs, hidden layer using 18 neurons and 25 neurons, 1 output) using matlab software.

Table 8. Data Training Backpropagation (18-18-25-1)

\begin{tabular}{|l|c|c|c|c|}
\hline \multirow{2}{*}{ types of logs } & \multirow{2}{*}{ Target } & \multicolumn{3}{|c|}{ ANN 18-18-25-1 } \\
\cline { 3 - 5 } & & Output & Error & SSE \\
\hline Agathis & 0,1010 & 0,1179 & $-0,017$ & 0,0002871012 \\
\hline Bakau & 0,1175 & 0,1220 & $-0,005$ & 0,0000206182 \\
\hline Bangkirai & 0,1123 & 0,1180 & $-0,006$ & 0,0000321676 \\
\hline
\end{tabular}




\begin{tabular}{|l|c|c|c|c|} 
Benuang & 0,1058 & 0,1181 & $-0,012$ & 0,0001519153 \\
\hline Damar & 0,1002 & 0,1173 & $-0,017$ & 0,0002911728 \\
\hline Duabanga & 0,1000 & 0,1171 & $-0,017$ & 0,0002924100 \\
\hline Indah & 0,1095 & 0,1169 & $-0,007$ & 0,0000553857 \\
\hline Jelutung & 0,1028 & 0,1166 & $-0,014$ & 0,0001915037 \\
\hline Kapur & 0,1426 & 0,1262 & 0,016 & 0,0002675318 \\
\hline Kruing & 0,1586 & 0,1314 & 0,027 & 0,0007402099 \\
\hline Meranti & 0,7436 & 0,7430 & 0,001 & 0,0000003975 \\
\hline Mersawa & 0,1167 & 0,1197 & $-0,003$ & 0,0000090746 \\
\hline Nyatoh & 0,1062 & 0,117 & $-0,011$ & 0,0001166199 \\
\hline Palapi & 0,1025 & 0,1164 & $-0,014$ & 0,0001933170 \\
\hline Ramin & 0,1107 & 0,1172 & $-0,006$ & 0,0000419061 \\
\hline Resak & 0,1011 & 0,1173 & $-0,016$ & 0,0002634024 \\
\hline Lainnya & 0,2961 & 0,1816 & 0,115 & 0,0131123455 \\
\hline Rimba Campuran & 0,2979 & 0,3418 & $-0,044$ & 0,0019248468 \\
\hline \multirow{2}{*}{} & & & Total & 0,0023394884 \\
\cline { 3 - 5 } & & MSE & 0,0001949574 \\
\hline
\end{tabular}

Table 9. Data Training Backpropagation (18-18-25-1)

\begin{tabular}{|c|l|c|c|c|c|c|}
\hline \multirow{2}{*}{ No } & \multirow{2}{*}{ types of logs } & \multirow{2}{*}{ Target } & \multicolumn{3}{|c|}{ ANN 18-18-25-1 } & \multirow{2}{*}{ Result } \\
\cline { 4 - 7 } & & & Output & Error & SSE & \\
\hline 1 & Agathis & 0,1004 & 0,1174 & $-0,017$ & 0,0002891225 & FALSE \\
\hline 2 & Bakau & 0,1026 & 0,1139 & $-0,011$ & 0,0001280527 & FALSE \\
\hline 3 & Bangkirai & 0,1205 & 0,1196 & 0,001 & 0,0000008642 & TRUE \\
\hline 4 & Benuang & 0,1028 & 0,1173 & $-0,015$ & 0,0002113321 & FALSE \\
\hline 5 & Damar & 0,1000 & 0,1172 & $-0,017$ & 0,0002958400 & FALSE \\
\hline 6 & Duabanga & 0,1000 & 0,1173 & $-0,017$ & 0,0002990691 & FALSE \\
\hline 7 & Indah & 0,1063 & 0,1183 & $-0,012$ & 0,0001450346 & FALSE \\
\hline 8 & Jelutung & 0,1001 & 0,1171 & $-0,017$ & 0,0002875826 & FALSE \\
\hline 9 & Kapur & 0,1144 & 0,1206 & $-0,006$ & 0,0000383658 & TRUE \\
\hline 10 & Kruing & 0,1598 & 0,1167 & 0,043 & 0,0018573919 & FALSE \\
\hline 11 & Meranti & 0,8605 & 0,8003 & 0,060 & 0,0036280287 & FALSE \\
\hline 12 & Mersawa & 0,1021 & 0,1170 & $-0,015$ & 0,0002235018 & FALSE \\
\hline 13 & Nyatoh & 0,1030 & 0,1176 & $-0,015$ & 0,0002140860 & FALSE \\
\hline 14 & Palapi & 0,1009 & 0,1173 & $-0,016$ & 0,0002676136 & FALSE \\
\hline 15 & Ramin & 0,1000 & 0,118 & $-0,018$ & 0,0003240000 & FALSE \\
\hline 16 & Resak & 0,1006 & 0,1171 & $-0,016$ & 0,0002720401 & FALSE \\
\hline 17 & Lainnya & 0,1937 & 0,2022 & $-0,008$ & 0,0000718748 & TRUE \\
\hline 18 & Rimba Campuran & 0,2048 & 0,1607 & 0,044 & 0,0019455050 & FALSE \\
\hline & & & & Total & 0,0074041859 & \multirow{2}{*}{$17 \%$} \\
\cline { 4 - 6 } & & & & MSE & 0,0006170155 & \\
\hline
\end{tabular}

In Table 9, the back-propagation architectural model 18-18-25-1 has an accuracy of 17\% with MSE: 0.0006170155 and epochs: 11442.

\subsection{Results}

By using the same parameters on each activation function, namely: sigmoid bipolar (tansig) with net.trainparam.epochs $=1500000$ net.trainparam.LR $=0.1$; net.trainParam.goal $=0.001$; net.trainParam.show $=1000$; Minimum error of 0.001-0.009 obtained result backpropagation architecture model 18-25-1 is the best with the accuracy of $72 \%$. Here is the comparison of 3 architectural models and the performance of each architecture model

Table 10. Data Training And Testing Backpropagation

\begin{tabular}{|l|l|l|l|}
\hline No & Architecture & Training & Testing \\
\hline
\end{tabular}




\begin{tabular}{|c|c|c|c|c|c|c|}
\hline & & Epoch & Time & MSE & MSE & Accuracy \\
\hline 1 & $18-18-1$ & 3797 & $00: 10$ & 0,0004756131 & 0,0026874460 & $6 \%$ \\
\hline 2 & $18-25-1$ & 660 & $00: 07$ & 0,0002685155 & 0,0221670942 & $72 \%$ \\
\hline 3 & $18-18-25-1$ & 11442 & $00: 15$ & 0,0001949574 & 0,0006170155 & $17 \%$ \\
\hline
\end{tabular}

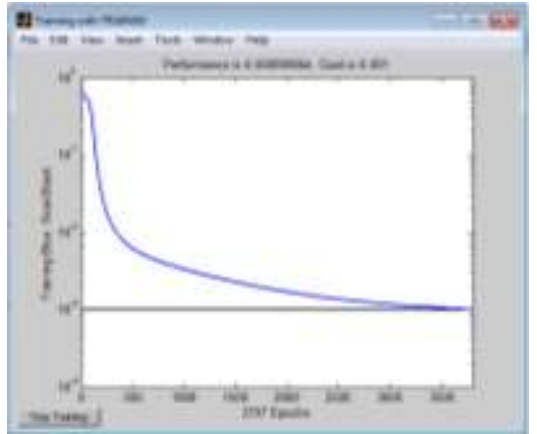

Figure 4. Performance of architecture model 18-18-1

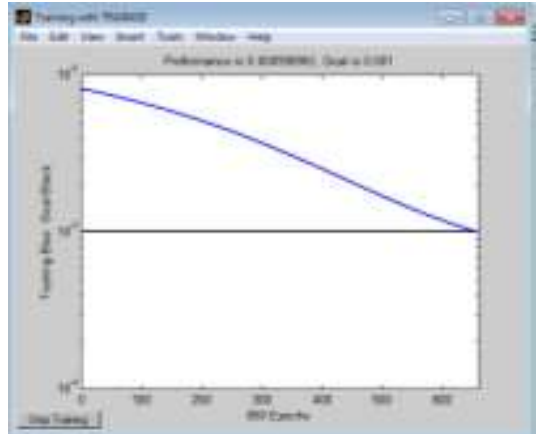

Figure 5. Performance of architecture model 18-25-1

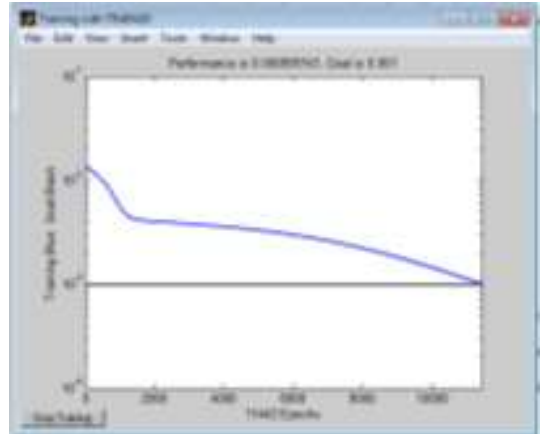

Figure 6. Performance of architecture model 18-18-25-1

\section{Conclution}

Based on the results of research in determining the architecture model on the prediction of the result of logs production using backpropogation method, obtained the result of 3 model architecture (18-18-1, 18-25-1 and 18-18-25-1) that model of architecture 18-25-1 is the best model with $72 \%$ accuracy.

\section{References}

[1] Sumijan, A. P. Windarto, A. Muhammad, and Budiharjo, "Implementation of Neural Networks in Predicting the Understanding Level of Students Subject," Int. J. Softw. Eng. Its Appl., vol. 10, no. 10, pp. 189-204, 2016.

[2] Che, Z.G., Chiang, T.A. and Che, Z.H., 2011. Feed-forward neural networks training: A comparison between genetic algorithm and back-propagation learning algorithm. International Journal of Innovative Computing, Information and Control, 7(10), pp.5839-5850.

[3] Choudhary, N.Y., Patil, M.R., Bhadade, U. and Chaudhari, B.M., 2013. Signature Recognition \& Verification System Using Back Propagation Neural Network. International Jorunal of IT, Engineering and Applied Sciences Research (IJIEASR), 2(1), pp.1-8.

[4] Baboo, S.S. and Shereef, I.K., 2010. An efficient weather forecasting system using artificial neural network. International journal of environmental science and development, 1(4), p.321.

[5] Che, Z.G., Chiang, T.A. and Che, Z.H., 2011. Feed-forward neural networks training: A comparison between genetic algorithm and back-propagation learning algorithm. International Journal of Innovative Computing, Information and Control, 7(10), pp.5839-5850.

[6] Choudhary, N.Y., Patil, M.R., Bhadade, U. and Chaudhari, B.M., 2013. Signature Recognition \& Verification System Using Back Propagation Neural Network. International Jorunal of IT, Engineering and Applied Sciences Research (IJIEASR), 2(1), pp.1-8.

[7] Shah, H., Ghazali, R., Nawi, N.M., Deris, M.M. and Herawan, T., 2013. Global artificial bee colony-Levenberq-Marquardt (GABC-LM) algorithm for 
classification. International Journal of Applied Evolutionary Computation (IJAEC), 4(3), pp.58-74.

[8] Castaño, B., Moreno, Á., Carbajo, M. and de Pedro, J., 2008. Artificial Intelligence and Bluetooth Techniques in a Multi-user M-learning Domain. IJCSA, 5(1), pp.113.

[9] Abubakar, A.I., Khan, A., Nawi, N.M., Rehman, M.Z., Wah, T.Y., Chiroma, H. and Herawan, T., 2016. Studying the Effect of Training Levenberg Marquardt Neural Network by Using Hybrid Meta-Heuristic Algorithms. Journal of Computational and Theoretical Nanoscience, 13(1), pp.450-460.

[10] Chiroma, H., Abdul-kareem, S., Ibrahim, U., Ahmad, I.G., Garba, A., Abubakar, A., Hamza, M.F. and Herawan, T., 2015. Malaria severity classification through Jordan-Elman neural network based on features extracted from thick blood smear. Neural Network World, 25(5), p.565.

[11] Husaini, N.A., Ghazali, R., Nawi, N.M., Ismail, L.H., Deris, M.M. and Herawan, T., 2014. Pi-Sigma Neural Network For A One-Step-Ahead Temperature Forecasting. International Journal of Computational Intelligence and Applications, 13(04), p.1450023.

[12] Nawi, N.M., Rehman, M.Z., Aziz, M.A., Herawan, T. and Abawajy, J.H., 2014, November. Neural network training by hybrid accelerated cuckoo particle swarm optimization algorithm. In International Conference on Neural Information Processing (pp. 237-244). Springer International Publishing.

[13] Nawi, N.M., Rehman, M.Z., Aziz, M.A., Herawan, T. and Abawajy, J.H., 2014, November. An Accelerated Particle Swarm Optimization Based Levenberg Marquardt Back Propagation Algorithm. In International Conference on Neural Information Processing (pp. 245-253). Springer International Publishing.

[14] M. Noor and H. Siregar, "Neural Network Analysis With Backpropogation In Predicting Human Development Index ( HDI ) Component by Regency / City In North Sumatera," I n te r n a ti o n a l Jo u rn a l Of I $n$ form a tion $S$ yst e $m$ Te c h n o logy, vol. 1, no. 1, pp. 22-33, 2017.

[15] Che, Z.G., Chiang, T.A. and Che, Z.H., 2011. Feed-forward neural networks training: A comparison between genetic algorithm and back-propagation learning algorithm. International Journal of Innovative Computing, Information and Control, 7(10), pp.5839-5850.

[16] Choudhary, N.Y., Patil, M.R., Bhadade, U. and Chaudhari, B.M., 2013. Signature Recognition \& Verification System Using Back Propagation Neural Network. International Jorunal of IT, Engineering and Applied Sciences Research (IJIEASR), 2(1), pp.1-8.

[17] Baboo, S.S. and Shereef, I.K., 2010. An efficient weather forecasting system using artificial neural network. International journal of environmental science and development, 1(4), p.321.

[18] Chiroma, H., Abdulkareem, S., Abubakar, A.I. and Herawan, T., 2014. Kernel functions for the support vector machine: comparing performances on crude oil price data. In Recent Advances on Soft Computing and Data Mining (pp. 273-281). Springer International Publishing.

[19] Hakim, R.F., Sari, E.N. and Herawan, T., 2014. Soft Solution of Soft Set Theory for Recommendation in Decision Making. In Recent Advances on Soft Computing and Data Mining (pp. 313-324). Springer International Publishing.

[20] Lasisi, A., Ghazali, R. and Herawan, T., 2014. Comparative performance analysis of negative selection algorithm with immune and classification algorithms. In Recent Advances on Soft Computing and Data Mining (pp. 441-452). Springer International Publishing. 


\section{Authors}

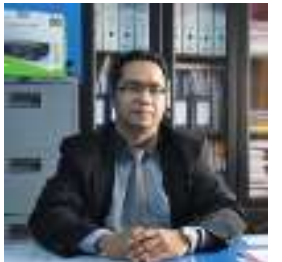

$1^{\text {st }}$ Author

Muhammad Noor Hasan

Universitas Graha Nusantara, Padangsidimpuan

noor.siregar@gmail.com 